\title{
Wideband Dipole Antennas with Parasitic Elements for Underwater Communications
}

\author{
O. Aboderin, L. M. Pessoa, H. M. Salgado \\ INESC TEC and Faculdade de Engenharia, Universidade do Porto Rua Dr. Roberto Frias, s/n \\ 4200-465 Porto, Portugal \\ (oluyomi.aboderin, luis.m.pessoa, hsalgado)@inesctec.pt,
}

\begin{abstract}
Propagation of underwater electromagnetic signals for real-time transmission and reception of data gathered during a survey mission between an Autonomous Underwater Vehicle (AUV) and underwater sensor nodes or a docking station, continues to generate a lot of interest worldwide. To this end, underwater antennas will play a significant role in ensuring good data rates and propagation distances for various underwater applications.

In this paper, the performance of two dipole antennas with different parasitic elements is assessed through simulation for usage in fresh water and operating in the High Frequency (HF) band. The antennas were designed in FEKO, an electromagnetic simulation software and their performance is assessed in terms of bandwidth, directivity and antenna-to-antenna distance. The results obtained show that these antennas exhibit wide bandwidth, which is important to achieve high data rates. Experimental results of the reflection coefficient of the fabricated antenna measured in fresh water are given that agree well with the simulation results.
\end{abstract}

Index Terms-Autonomous Underwater Vehicle; Underwater Antennas; dipole antennas; parasitic elements; directivity; bandwidth; FEKO.

\section{INTRODUCTION}

There has been global attention to the underwater communication technology both from the industry and the scientific community, which is due to the wide range of applications that this mean of communication covers, which includes: coastline protection and surveillance, off-shore oil and gas field monitoring, underwater environmental observation for exploration, oceanographic data collection. Here we target specific applications which will require data exchange between two or more Autonomous Underwater Vehicles (AUVs) or Remotely Operated Vehicles (ROVs) [1], [2]. This demands enough bandwidth for high data rates, which may be required for real-time video exchange between the underwater bodies and these can be either a directional or omnidirectional scenario.

In underwater communications, the three widely used communication technology for underwater applications are acoustics signals, optical signals and Electromagnetic (EM) signals [3]. The acoustic and ultrasonic systems guarantee long range communications (up to tens of kilometers), but this technology suffers from; poor immunity to noise, low data-rates and high latency and is therefore unfit for realtime and broadband underwater communications [2], [4], [5]. Optical communication links on the other hand allows high data-rates and low latency, which are important advantages over acoustics technology. However, optical technologies are affected by marine fouling and suspended particles in water and most importantly require very good alignment. The third technology in line with underwater communication is electromagnetic (EM) communications [4], [5]. Propagation of EM signals in water, neither require strong alignment nor are affected by water condition and the technology can deliver data rates up to $10 \mathrm{Mbps}$, though at very short distances, due to signal attenuation, as it increases with increasing operating frequency. Thus, effort must be made to design antennas that can operate at seemingly low frequencies [6], [7]. Based on these requirements, the dipole antennas with parasitic elements designed and presented in this paper achieved up to $70 \mathrm{MHz}$ bandwidth between $10 \mathrm{MHz}$ and $80 \mathrm{MHz}$.

The rest of this paper is organised as follows: section II presents the propagation of electromagnetic waves in the media; in section III the design of the underwater antennas is presented with two subsections dedicated to the design for usage in fresh water and for sea water respectively; simulation results and interpretations are given in section IV, and the conclusions are addressed in section $\mathrm{V}$.

\section{Electromagnetic Wave Propagation in Water}

An important consideration in designing underwater antennas is the conductivity of water, where conductivity in fresh water ranges from $0.005 \mathrm{~S} / \mathrm{m}$ to $0.05 \mathrm{~S} / \mathrm{m}$, whereas in sea water the value is $4 \mathrm{~S} / \mathrm{m}$. This is an important parameter as it determines the attenuation at the desired frequency [4], [5]. The propagation constant, $\gamma$ in a conducting medium is given by $[8]$

$$
\gamma=\sqrt{j \omega \mu(j \omega \epsilon+\sigma)}=\alpha+j \beta
$$

Here, $\omega$ is the angular frequency measured in radian per second, $\mu$ is the absolute permeability $\left(\mu=\mu_{0} \mu_{r}\right), \epsilon$ is the absolute permittivity $\left(\epsilon=\epsilon_{r} \epsilon_{0}\right), \sigma$ is the conductivity measured in Siemens per metre, $\alpha$ is the attenuation constant measured in Neper per metre and $\beta$ is the phase constant in radians per unit length. In [9] the propagating medium has been classified into two types, namely the dielectric media or conducting media. The medium is a dielectric or insulator, when $\sigma / \omega \epsilon<<1$, which means the displacement current is much greater than the conductive current. Conversely, when $\sigma / \omega \epsilon>>1$, the media is considered to be a good conductor, meaning the conductive 


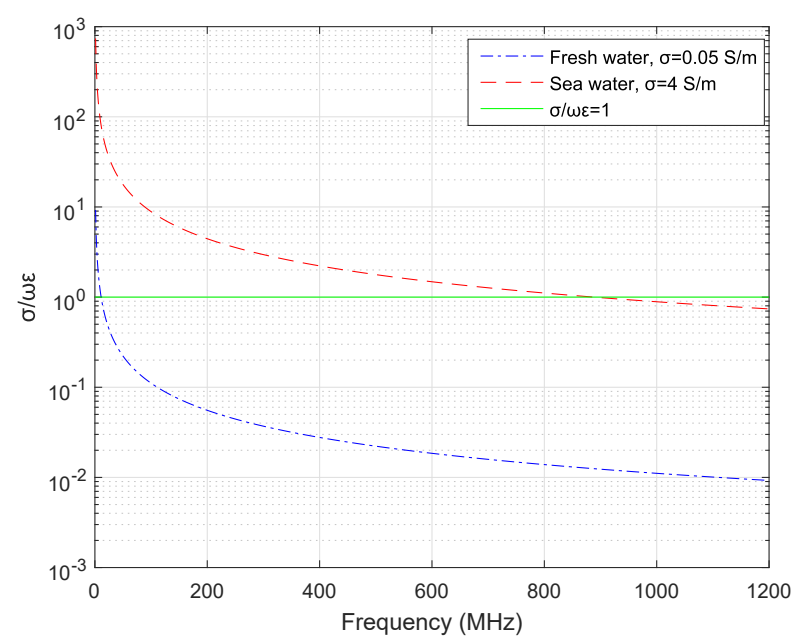

Fig. 1. Behavior of $\sigma / \omega$ as a function of frequency in fresh and sea water [10]

current is much greater than the displacement current. It is based on these analyses that the dependence of $\sigma / \omega \epsilon$ as a function of operating frequency for fresh and sea water is presented in Fig. 1. From the plots, fresh water behaves as a conductor for frequencies below $10 \mathrm{MHz}$, it thereafter behaves as a dielectric media, whereas the change from conducting to dielectric media occurs at $888 \mathrm{MHz}$ in sea water. This simply means that for lower frequencies, sea water behaves as a good conductor.

The expression for $\alpha$ and $\beta$ are deduced from equation (1) and are given as equation (2) and (3) respectively [2].

$$
\begin{aligned}
& \alpha=\omega \sqrt{\mu \epsilon} \sqrt{1 / 2\left(\sqrt{1+(\sigma / \omega \epsilon)^{2}}-1\right)} \\
& \beta=\omega \sqrt{\mu \epsilon} \sqrt{1 / 2\left(\sqrt{1+(\sigma / \omega \epsilon)^{2}}+1\right)}
\end{aligned}
$$

Equation (2) and (3) are vital for understanding the attenuation at various operating frequencies and in the determination of the best operating frequency of the antennas. The attenuation of an electromagnetic waves as a function of frequency is presented in Fig. 2. It is observed from the plots that attenuation is relatively lower at frequencies up to hundreds of Mega Hertz in freshwater. Yet, in the sea water, the attenuation increases with increasing frequency, in the range of $1 \mathrm{MHz}$ to $1 \mathrm{GHz}$. Thus, the antennas in this work are therefore designed to operate at lower frequency.

\section{OVERVIEW OF THE ANTENNAS AND DESIGNING}

In one of the previous works for underwater antenna design in this group [11], the capability of J-pole antenna configurations to achieve bandwidths above $30 \mathrm{MHz}$ was assessed and presented. However, when there is the need for higher throughput, for example, as in real-time video transmission, which may occur between underwater sensor nodes and an AUV or between AUVs and a docking station, these antennas

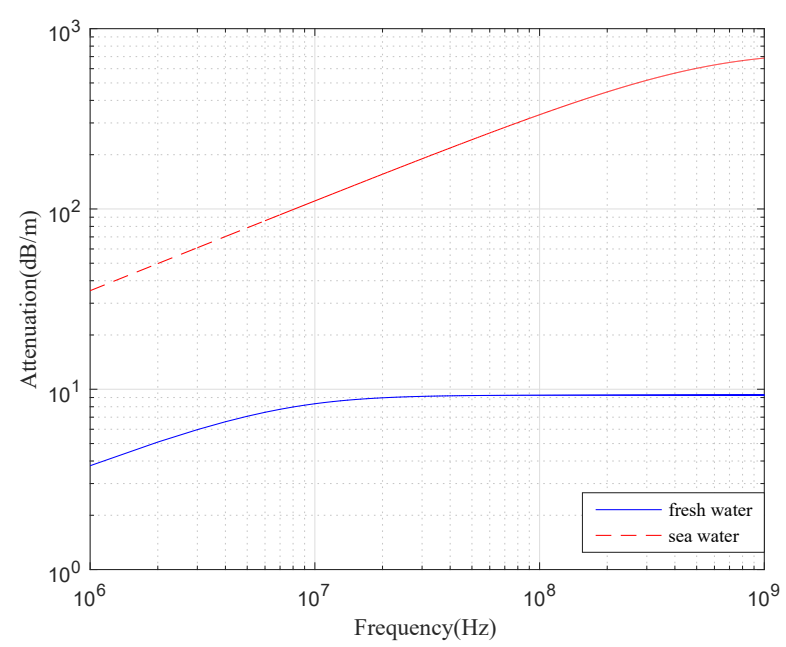

Fig. 2. Attenuation of an electromagnetic wave propagating in fresh and sea water

might not be able to sustain such data rates. It is therefore expediently important to construct underwater antennas that will meet the bandwidth requirements for such defined operations. Thus, in this paper, the performance of dipole antennas with two different parasitic elements was assessed in fresh water and measured in terms of bandwidth and directivity. The two forms of dipoles are: the dipole antenna with ordinary parasitic element (DipP) and the dipole antenna with a 'coily' (the parasitic element containing a coil) parasitic element (DipH).

The models of these antennas, as designed in FEKO software, are presented in Fig. 3. The two antennas are halfwave dipoles and to calculate their respective dimensions, the wavelength is given by:

$$
\lambda=2 \pi / \beta
$$

The spacing of the parasitic elements to the antennas was carefully considered during the design of the antennas. From the simulation, it is observed that the closer the parasitic element to the dipole, the higher the bandwidth, but this comes at the expense of reduce effciency. This is because the current flowing in the parasitic elements flows in opposite direction to the one flowing in the dipole, thereby reducing the efficiency of the antennas. Thus, after several parametric analysis in which both the antenna efficiency and the bandwidth were duly considered, the elements were placed at a distance of $2.5 \mathrm{~cm}$ from the antennas. The dimensions pf the antennas is presented in Table I, where D1 is the half wavelength element of the dipole arms for the two antennas, D2 is the quarter wavelength element of the parasitic arms of DipP antenna, D3 is the length of the coil added in DipH, and D4 is the $\lambda / 16$ elements attached to the two ends of the coil. The dimension of this coil is calculated based on the analysis of helical antennas presented in [12], [13]. The thickness of the wires used in the design of the antennas is $3 \mathrm{~mm}$ and they are covered 


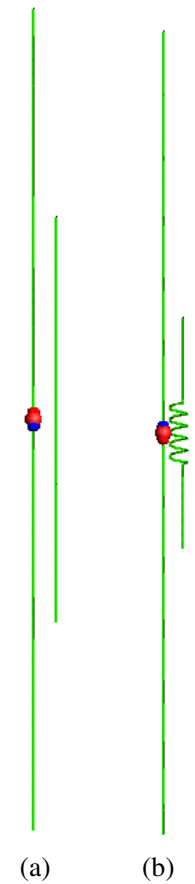

Fig. 3. (a) Dipole with ordinary parasitic element (b) Dipole with coily parasitic element

TABLE I

DIMENSION OF THE ANTENNAS

\begin{tabular}{|c|c|c|c|c|}
\hline S /No. & D1 & D2 & D3 & D4 \\
\hline 1 & 475.00 & 237.50 & 72.00 & 82.75 \\
\hline
\end{tabular}

with insulating material with thickness of $50 \mu \mathrm{m}$ and relative permittivity of 3 .

\section{Simulation Results}

The two antennas were simulated in fresh water scenario and the results corresponding to the reflection coefficient as a function of frequency for the antennas are presented in Fig. 4. In this respect at $-10 \mathrm{~dB}$ of the reflection coefficient, DipP achieved $65 \mathrm{MHz}$ bandwidth, between $10 \mathrm{MHz}$ and $74 \mathrm{MHz}$, whereas DipH has a slightly higher bandwidth of $74 \mathrm{MHz}$, between $9 \mathrm{MHz}$ and $83 \mathrm{MHz}$. These results shows an improvement when compared with the performance of the J-pole antenna in the same medium, as presented in [11]. Another important parameter that was simulated is the directivity which, at $25 \mathrm{MHz}$ equals $10.70 \mathrm{~dB}$ and $12.50 \mathrm{~dB}$ and at $40 \mathrm{MHz}$ equals $8.50 \mathrm{~dB}$ and $9.92 \mathrm{~dB}$ for DipP and DipH respectively. The radiation pattern of the antennas in E-plane, at $25 \mathrm{MHz}$ and $40 \mathrm{MHz}$ are presented in Fig. 5. It is clearly seen from the plots that at lower frequency, they have similar behaviour and thus their pattern overlap. But at $40 \mathrm{MHz}$, DipP is beginning to develop side lobes whereas pattern for DipH though narrower than what was obtained for the same antenna at $25 \mathrm{MHz}$, but no feasible side lobe seen on it. Similarly the radiation pattern in H-plane at the same frequencies are given in Fig. 6. Here, it seen that the pattern

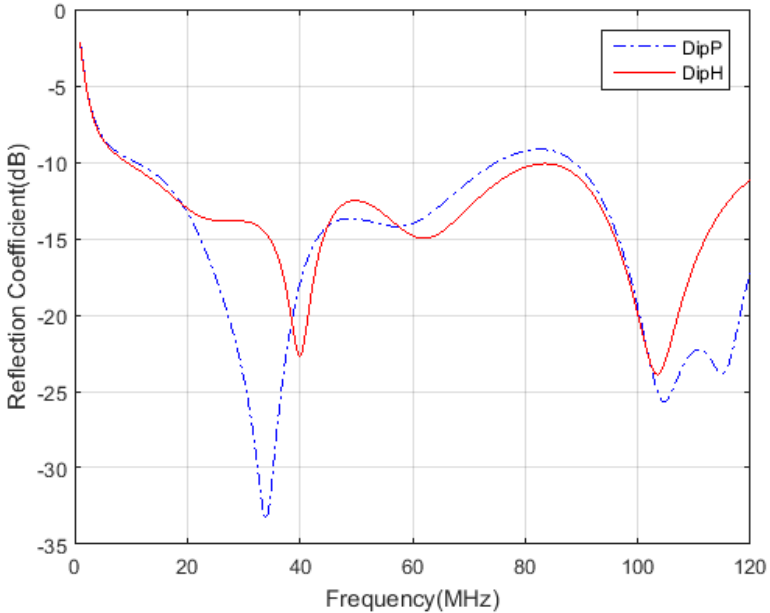

Fig. 4. Reflection coefficient against frequency for the two antennas

shows that the present of parasitic changed the shape of the plane when compared with the results obtained and presented in [10]. These are important results both for the data rates and the propagation distance.

Also measured is the $S_{21}$ as a function of distance between two antennas of each type (that is distance between two of DipP antennas and two of DipH antennas) at $25 \mathrm{MHz}$ and 40 Mhz respectively, the results are presented in 7 . The behaviour of the two antennas are similar in this respect, as the plots seemingly overlap at the considered frequencies. Considering a transmission system operating in this medium, with a receiver having a sensitivity of $90 \mathrm{dBm}$ and a transmitter with an output power of $10 \mathrm{dBm}$, the maximum expected range of operation is around $7 \mathrm{~m}$ at $25 \mathrm{MHz}$ and $6.4 \mathrm{~m}$ at $40 \mathrm{MHz}$, this is similar to the results presented in [10]. In all, the dipole with coily parasitic element yielded higher performance than the one with ordinary parasitic element. However, for reasons concerned with easee of fabrication and precision of alignment of the elements, only the dipole antenna with ordinary parasitic element was manufactured and tested accordingly, to validate the simulated results with experimental measurements.

\section{EXPERIMENTAL RESUlTS}

The dipole antenna with ordinary parasitic element was manufactured using the dimension specified in section III. The thickness of copper wires used is $3 \mathrm{~mm}$ and current baluns were added to the antennas to reduce undesired radiation on the coaxial cables that could change some of the charateristics of the antennas by interfering with the measured parameters. Thereafter, the measurements was set up in a freshwater pool located at INESC TEC, with the antennas at a depth of $2.5 \mathrm{~m}$ from the surface and placed on the centre of the tank (which has dimensions of $10 \mathrm{~m} \times 6 \mathrm{~m} \times 5.5 \mathrm{~m}$ and the conductivity of water was $0.0487 \mathrm{~S} / \mathrm{m}$ at $25^{\circ} \mathrm{C}$ ). Pictures of the antenna and the measurement setup are given in Fig.8. The simulation results of the reflection coefficient are compared with the measured results in Fig.9. The measured reflection coefficient 


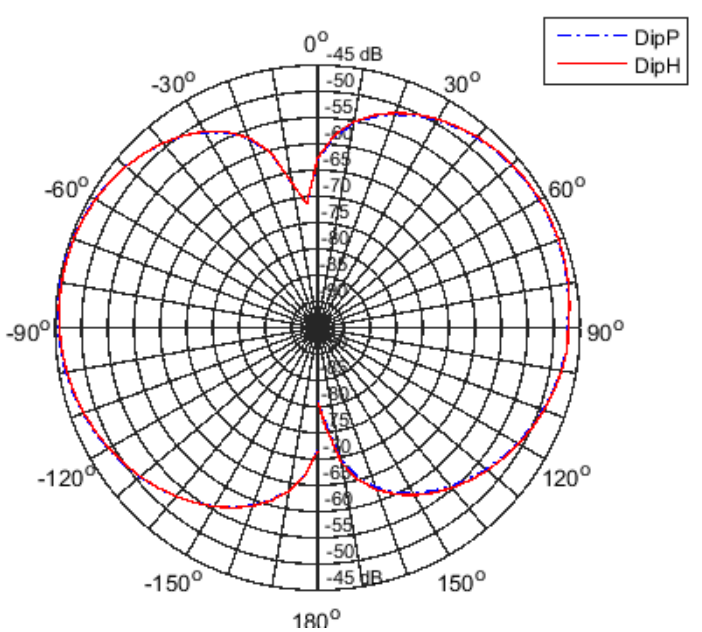

(a)

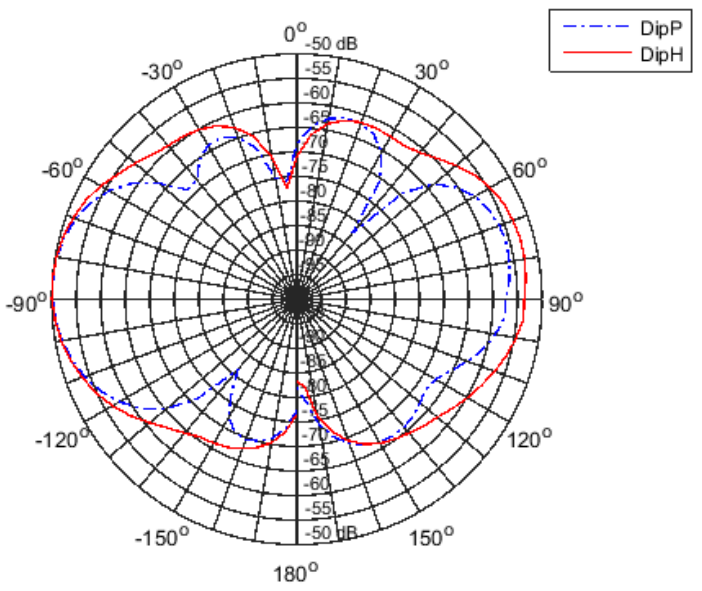

(b)

Fig. 5. Radiation pattern of the antennas in E-plane at (a) $25 \mathrm{MHz}$ and (b) $40 \mathrm{MHz}$

agrees well with the simulation, except for a shift of the resonance frequency to $44 \mathrm{MHz}$ confirming that the antenna is a wideband. The difference in magnitude of the resonance between the simulated and the measured results is attributed to the attenuation through the cable.

Similarly the radiation pattern of the antenna, in the E-plane was also measured experimentally in the water at $25 \mathrm{MHz}$ and $40 \mathrm{MHz}$. Results are shown in Fig. 10. It is seen that the results match quite well.

\section{CONCLUSION}

The performance of two dipole antennas with different parasitic elements has been analyzed through simulation, in terms of their respective bandwidth, directivity, and their $S_{21}$ as a function of distance between two antennas of the same type. The spacing between the parasitic elements and the antennas must be carefully considered to ensure the efficiency is not affected, while improving the bandwidth. The two antennas are the dipole with an ordianry parasitic element (DipP) and dipole with a coily parasitic element (DipH)

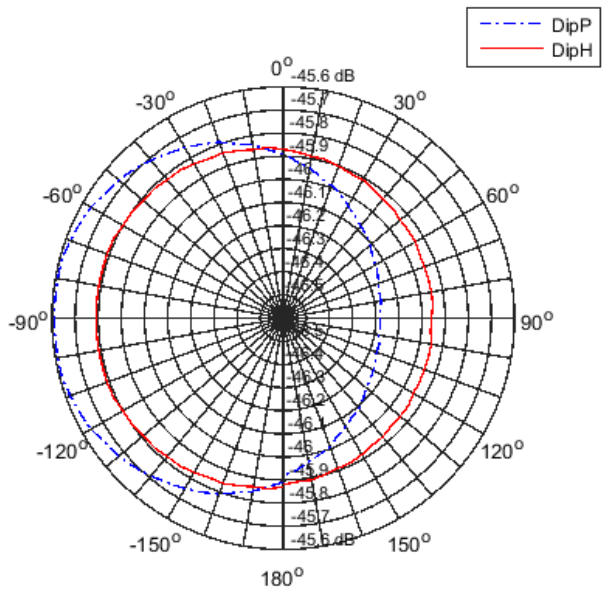

(a)

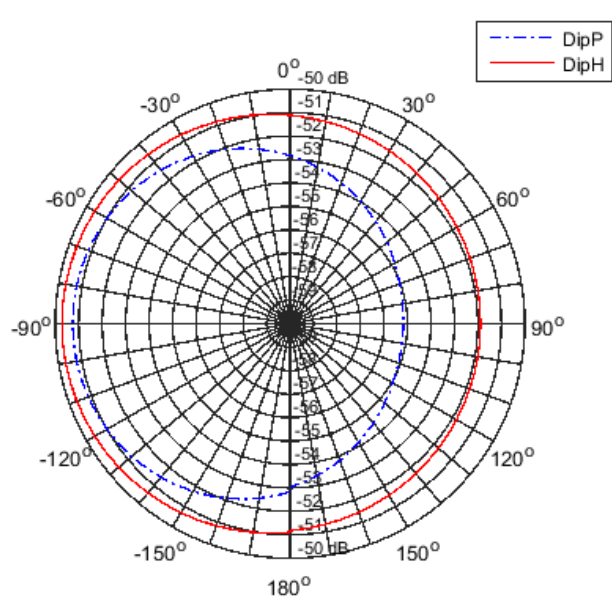

(b)

Fig. 6. Radiation pattern of the antennas in H-plane at (a) $25 \mathrm{MHz}$ and (b) $40 \mathrm{MHz}$

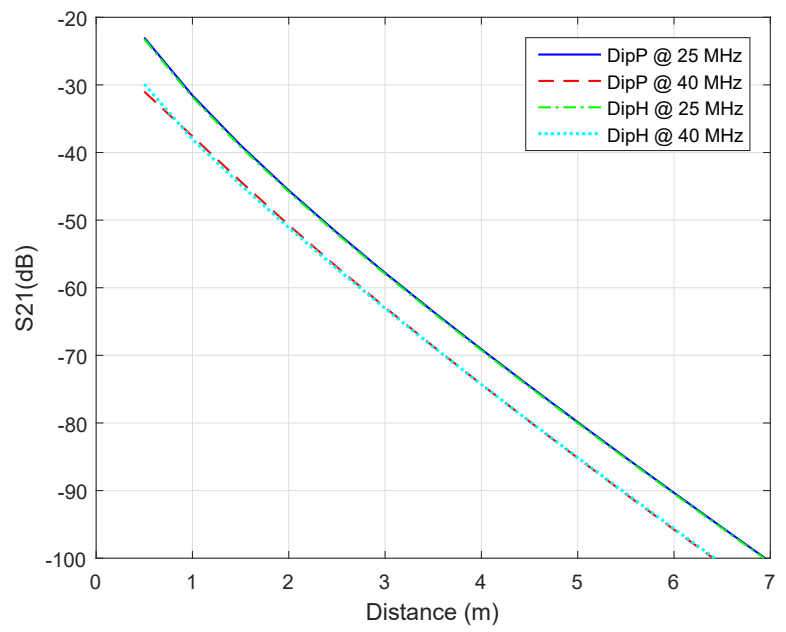

Fig. 7. $S_{21}$ as a function the distance between two antennas for both DipP and DipH 


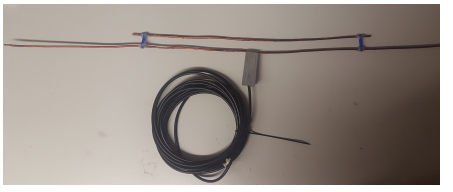

(a)

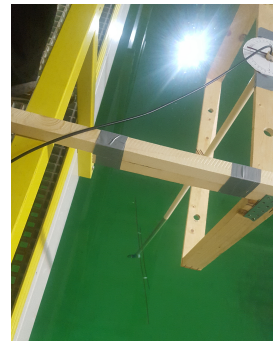

(b)
Fig. 8. Photographs of the DipP antenna after fabrication and during measurement

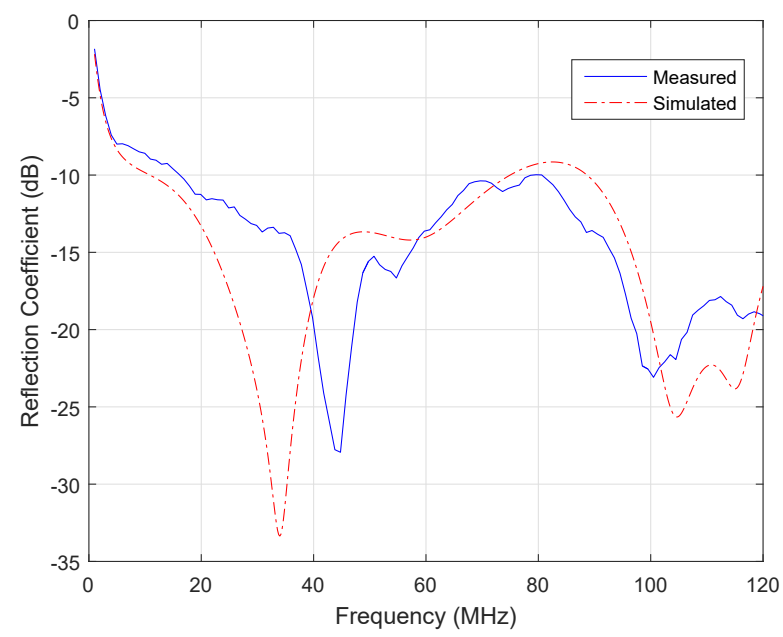

Fig. 9. Simulation versus measurement for the DipP Antenna

respectively. These antennas exhibit wideband charateristics, with DipH having slightly higher bandwidth and directivity than DipP, only in their $S_{21}$ that the two antennas has no noticeable differences at $25 \mathrm{MHz}$ and $40 \mathrm{MHz}$. Despite these slight advantages of DipH, DipP was manufactured for ease of fabrication.

In addition, experimental work was carried out in large fresh water tank and the bandwidth obtained for the manufactured antenna are in good agreement with the simulation results. The radiation pattern was only presented in E-plane at $25 \mathrm{MHz}$ and $40 \mathrm{MHz}$ respectively and these also are in good agreement with the simulation results. In conclusion, the achieved bandwidth and directivity are important for underwater applications when considering the data rates and the propagation distance between two underwater bodies. Finally, futher work will be considered in fabrication of the dipole antenna with coily parasitic element for validating the simulation results with measurement.

\section{ACKNOWLEDGEMENT}

This work was supported by integrated project CORAL - "NORTE-01-0145-FEDER-000036", which is financed by the North Portugal Regional Operational Programme (NORTE 2020), under the PORTUGAL 2020 Partnership Agree-

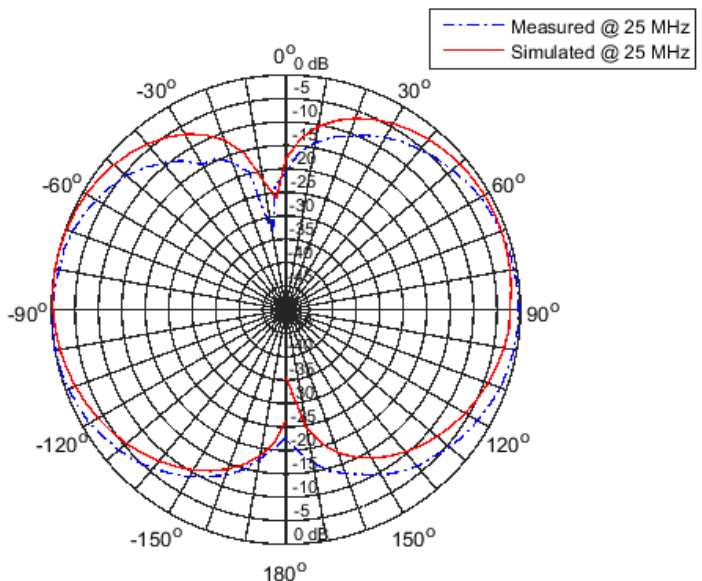

(a)

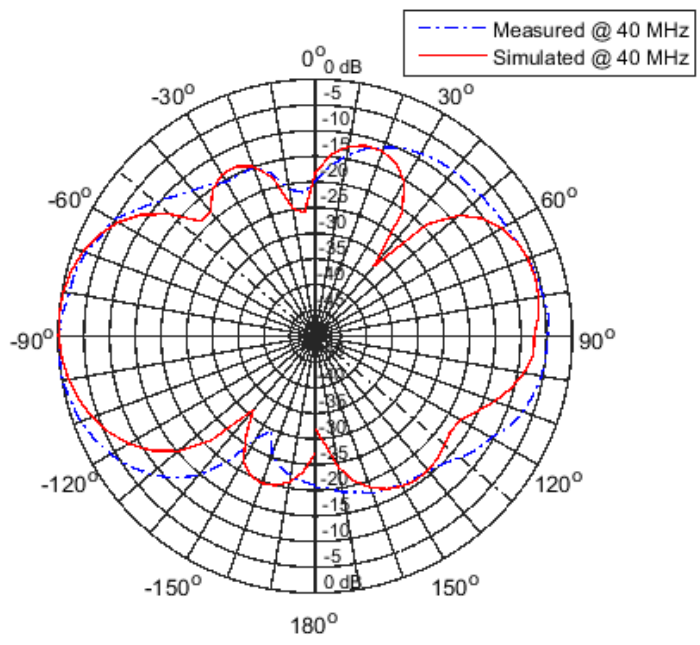

(b)

Fig. 10. Simulation versus measurement of the 2-D radiation pattern in Eplane at (a) $25 \mathrm{MHz}$ and (b) $40 \mathrm{MHz}$

ment, and through the European Regional Development Fund (ERDF). The work also contributed to project ENDURE Enabling Long-Term Deployments of Underwater Robotic Platforms in Remote Oceanic Locations (PT02 Aviso4 0015). ENDURE benefits from a $218157 €$ grant from Iceland, Liechtenstein and Norway through the EEA Grants. The support of STRONGMAR: STRengthening MARritime technology Research Center (H2020-TWINN-2015 (CSA)-692427) and the TEC4SEA research infrastructure (www.tec4sea.com) is acknowledged.

\section{REFERENCES}

[1] H. F. G. Mendez, F. Le Pennec, C. Gac, and C. Person, "High performance underwater uhf radio antenna development," IEEE OCEANS Conference Jun 06-09, 2011 Santander, SPAIN.

[2] S. Jiang and S. Georgakopoulos, "Electromagnetic wave propagation into fresh water," International Conference on Detection and Classification of Underwater Targets, France, p. 6, 2012.

[3] J. Lloret, S. Sendra, M. Ardid, and J. J. P. C. Rodrigues, "Underwater wireless sensor communications in the $2.4 \mathrm{GHz}$ ISM frequency band," Sensors, vol. 12, no. 4, pp. 4237-4264, 2012. 
[4] J. H. Goh, A. Shaw, and A. I. Al-Shamma'a, "Underwater wireless communication system," Journal of Physics, p. 6, 2009.

[5] H. Zhang, D. Geng, G. Zhang, and T. A. Gulliver, "The impact of antenna design and frequency on underwater wireless communications," IEEE Pacific Rim Conference on Communications, Computers and Signal Processing (Pacrim), pp. 868-872, univ Victoria, Victoria, CANADA 2011.

[6] N. George, G. R., and D. C. N, "Design of wide band antenna for ocean communication: Review," International Journal of Advanced Computer Research, vol. 4, no. 14, p. 8, 2014.

[7] E. A. Karagianni, "Electromagnetic waves under sea: Bow-tie antenna design for wi-fi underwater communications," Progress In Electromagnetics Research, vol. 41, p. 10, 2015.

[8] K. P. Hunt, J. J. Niemeier, and A. Kruger, "Short paper: Antennas for mussel-based underwater biological sensor networks in rivers," p. 4, 2010.
[9] K. C. David, "Field and wave electromagnetics," IEE Proceedings- $a$ Science Measurement and Technology, vol. 131, no. 8, pp. 660-660, 1984.

[10] S. I. Inacio, M. R. Pereira, H. M. Santos, L. M. Pessoa, F. B. Teixeira, M. J. Lopes, O. Aboderin, and H. M. Salgado, "Antenna design for underwater radio communications," Oceans2016, Shanghai China, p. 5, 2016.

[11] O. Aboderin, S. I. Inácio, H. M. Santos, M. R. Pereira, L. M. Pessoa, and H. M. Salgado, "Analysis of j-pole antenna configurations for underwater communications," Oceans2016, Monterey Califonia, p. 5, 2016.

[12] A. R. Djordjevic, A. G. Zajic, M. M. Ilic, and G. L. Stuber, "Optimization of helical antennas," IEEE Antennas and Propagation Magazine, vol. 48, no. 6, pp. 107-115, 2006

[13] C. A. Balanis, Antenna Theory Analysis and Design, 3rd ed. New Jersey, USA: John Wiley \& Sons, 2005. 